LAWRENCE LIVERMORE N A T IO N A L LABORATORY

\title{
Dynamics of Collisions Revealed by Classical Methods
}

R. E. Olson, J. Fiol, J. Perez, P. Beiersdorfer

October 19, 2004

8th Workshop on Fast Ion-Atom Collisions

Debrecen, Hungary

September 1, 2004 through September 3, 2004 
This document was prepared as an account of work sponsored by an agency of the United States Government. Neither the United States Government nor the University of California nor any of their employees, makes any warranty, express or implied, or assumes any legal liability or responsibility for the accuracy, completeness, or usefulness of any information, apparatus, product, or process disclosed, or represents that its use would not infringe privately owned rights. Reference herein to any specific commercial product, process, or service by trade name, trademark, manufacturer, or otherwise, does not necessarily constitute or imply its endorsement, recommendation, or favoring by the United States Government or the University of California. The views and opinions of authors expressed herein do not necessarily state or reflect those of the United States Government or the University of California, and shall not be used for advertising or product endorsement purposes. 


\title{
Dynamics of Collisions Revealed by Classical Methods
}

\author{
R. E. Olson ${ }^{1}$, J. Fiol ${ }^{2}$, J. Perez ${ }^{3}$ and P. Beiersdorfer ${ }^{4}$ \\ ${ }^{1}$ Physics Department, University of Missouri-Rolla, Rolla, MO 65401 USA \\ ${ }^{2}$ Centro Atómico Bariloche, Río Negro, Argentina \\ ${ }^{3}$ Physics Department, Luther College, Decorah, Iowa 52101 USA \\ ${ }^{4}$ Physics Division, Lawrence Livermore National Laboratory, Livermore, CA 94551 USA
}

\begin{abstract}
Single ionization of helium by impact of $3.6 \mathrm{MeV} / \mathrm{u} \mathrm{Au}^{53+}$ ions is investigated by means of quantum and classical methods. Calculation of fully-differential cross sections are compared to recently published data for ionization of low-energy electrons as a function of the momentum transferred by the projectile to the target system. The results show that inclusion of the resolution and uncertainties present in the experiment has a major influence on both the shape and magnitude of the calculated cross sections. The effect of using a two-electron model for the He target along with including electron-electron correlation are also investigated. However, after incorporating all the experimental conditions within the calculations, the one- and two-electron results present similar behavior.
\end{abstract}

Keywords: Ion-Atom Collisions

PACS numbers: $34.10+x, 34.50 . \mathrm{Fa}$

\footnotetext{
${ }^{1}$ Present address: Centro Atómico Bariloche (8400) Río Negro, ARGENTINA.

E-mail: fiol@cab.cnea.gov.ar
} 


\section{Introduction}

In this paper we investigate helium single ionization by collision with $3.6 \mathrm{MeV} / \mathrm{u} \mathrm{Au}^{53+}$ ions

$$
\mathrm{Au}^{53+}+\mathrm{He} \rightarrow \mathrm{Au}^{53+}+\mathrm{He}^{+}+\mathrm{e}
$$

The general problem of atomic ionization by impact of fast heavy ions has recently raised considerable interest [1-5]. The availability of recent experimental data obtained in kinematically complete experiments [6], where all the independent components of the particles' final momenta are measured, has provoked considerable effort. Due to the detail of the data, comparison to these fully-differential cross-sections are regarded as a stringent test of three-body theories.

In particular, atomic ionization by impact of highly charged $\mathrm{Au}^{53+}$ has proved to be remarkably difficult to describe theoretically due to the long range of the projectile interaction. High-order theories reproduce accurately the angle and energy spectra of the ionized electrons [7]. However, the situation is different for cross sections related to the projectile deflection. In particular, for single ionization double differential cross sections $\mathrm{d} \sigma / \mathrm{dE}_{\mathrm{e}} \mathrm{dQ}_{\text {perp }}$, the comparison between theory and experiment has shown poor agreement [2]. Although important discrepancies observed were first attributed to the lack of a correct internuclear interaction in the theoretical treatment, its inclusion has not improved the accord with the measured data $[1,8]$. Cross sections calculated with different approximations, including the classical trajectory Monte Carlo (CTMC) and quantummechanical Continuum Distorted Wave (CDW) theories, that include the correct internuclear interaction, have shown a qualitatively different behavior than that observed in the experimental data. Remarkably, these state-of-the-art classical and quantum mechanical approaches show excellent agreement with each other $[1,3]$ but yield double 
differential cross sections that are much smaller than the experimental data for large momentum transfer.

There may be various reasons for the significant discrepancies observed between theory and experiment. On one hand, the simplification of the problem to a pure three-body collision neglects any two-electron transitions such as double ionization or excitation-ionization processes. For instance, double-ionization has been shown to be more important than single ionization for large values of the momentum transfer [1]. Moreover, the use of a model to describe the interactions of the $\mathrm{He}^{+}$core with both the electron and the projectile introduces additional ambiguities.

In this work we discuss some of the difficulties found in the determination of helium ionization cross sections. We address the problem in two different steps. In the first part, a three-body collision model with realistic Hartree-Fock target interactions is employed in both the CTMC and CDW theories. These models improve the target description over previous calculations $[1,2,6,8]$. Later, the effects of many-bodies are studied by including different two-electron models of the target atom in the classical calculations.

\section{Theory}

The three-body models employed in the quantum-mechanical and classical theories are essentially equivalent. The present CDW calculations are an extension of those presented previously for hydrogen-like targets [3,9]. As an extension to the previous papers, the bound initial and continuum final states of the He system are described as eigenfunctions

of the same Hartree-Fock potential [10]. Here, the $\mathrm{He}^{+}$core interaction with the active electron is modeled by the two-parameter potential [11]

$$
r \mathrm{~V}(r)=-1-1.48 /[(\exp (2.63 r)-1]
$$


The present three-body CTMC calculations were performed using an initial state described by means of a Wigner distribution. The method uses a linear combination of microcanonical atomic states [12] such that the initial distribution resembles closely the He quantum-mechanical density probabilities both in position and momentum space [13]. The initial state and the evolution of the system were computed under the hypothesis that the $\mathrm{He}^{+}$core can be described as a structureless particle. The central potential employed to model the $\mathrm{He}^{+}$core is the same as in the CDW calculations.

We note that the three-body treatments used in the CDW and CTMC methods differ slightly. While the classical theory includes the interaction of the recoil-ion with both the projectile and the electron in the form of a Hartree-Fock potential at all times, in the present CDW theory the projectile-target interaction is approximated by a Coulomb potential with the target charge set equal to unity. However, within the scope of the present work this approximation is justified.

In order to test the validity of a three-body approach for the ionization of a two-electron atom we also performed four-body CTMC calculations. We have employed the so-called $d$-CTMC method that dynamically incorporates the electron-electron interaction when the atom is perturbed by the projectile $[1,13]$. Additionally, we compare the results with a corrected $d$-CTMC description that includes radial and angular correlation in the initial state. For this latter model the two electrons are completely correlated by initially positioning them on opposite sides of the nucleus with opposite momenta.[JF2] In both $d$-CTMC theories Wigner distributions identical to the threebody case have been used for the initial state. In the model without correlation, each electron is set independently in a similar form than in the three-body calculations. In the correlated version of the $d$-CTMC only one electron is randomly determined. 


\section{Results and discussion}




\section{Ionization into the scattering plane}

Figure 1 shows plots of triple differential cross-sections (TDCS) $\mathrm{d} \sigma / \mathrm{dE} \mathrm{el}_{\mathrm{el}} \mathrm{d} \Omega_{\mathrm{el}} \mathrm{d} Q_{\text {perp }}$ as a function of the electron energy $\mathrm{E}_{\mathrm{e}}$ and $Q_{\text {perp }}$, the component of the momentum transferred by the projectile to the target system in the direction perpendicular to the incident velocity $\boldsymbol{v}$, atomic units are used for all the cross sections. The TDCS obtained with the $\mathrm{CDW}$ approximation are presented as a function of the polar electron angle $\theta_{\mathrm{el}}$ for emission of $10 \mathrm{eV}$ electrons in the scattering plane defined by the incident projectile velocity and the final momentum transfer vector (azimuthal angle $\varphi_{\mathrm{el}}=0^{\circ}$ or $180^{\circ}$ ). Shown are plots for momentum transfers of 0.45 and 0.65 a. u., respectively.

The angular distributions shown in Fig. 1 present a maximum along the direction of the momentum transfer $\boldsymbol{Q}$, which is in the range $\theta_{\mathrm{el}} \approx 60^{\circ}-70^{\circ}$. This result is consistent with the idea of a strong binary projectile-electron collision with no participation by the recoil-ion. One can also observe in Fig. 1 a small maximum in the direction that is the mirror angle to that of the momentum transfer $\boldsymbol{Q}$. The production of these so-called "swing by" electrons has been predicted recently for ionization of hydrogen by $\mathrm{Au}^{53+}$ impact [3]. It was shown that the mechanism which produces these electrons is only possible for impact of highly charged ions. Finally, note that the absolute magnitude of the cross sections strongly decreases with increasing momentum transfer.

Figure 2 shows the CDW and CTMC cross sections averaged over bins of momentum transfer $Q$ as used in the analysis of the experimental data. The classical results were calculated using the following bin sizes: energy $E_{e}= \pm 3 \mathrm{eV}$, and angular acceptances $\theta_{\mathrm{el}}= \pm 5^{\circ}$ and $\varphi_{\mathrm{el}}= \pm 10^{\circ}$. These values are the same as those reported for the available experimental data [2,6]. Good agreement is observed between CDW and 
CTMC results but neither of them resemble the measured cross sections of Fisher et al [6].

\section{Comparison with experimental data}

The absolute values of the differential cross sections for single ionization strongly decrease with increasing momentum transfer. As shown in Fig. 2 the absolute magnitudes are strongly influenced by the experimental momentum and angular resolution. In the cross sections of Fig. 2 the theoretical results were averaged over bins whose size were the same as those reported for the experimental data. However, the acceptance bins are not the only resolution parameters that must be taken into account in the comparison with experimental cross sections.

It is important to discern between the acceptance parameters of the experiment given by the bin sizes employed in the analysis of the data (as described above) and the uncertainties in the measurement of the particles' momenta, related to the actual experimental conditions. In figure 3 we present the CDW and CTMC results corrected by considering some of the uncertainties of the measured data $[6,14]$. The one standard deviation experimental uncertainty in the determination of the momentum of the recoil ion is approximately \pm 0.22 a. u. for the component perpendicular to the incident velocity $\mathbf{v}$ and \pm 0.1 a. $u$. in the parallel component. For the electron, the uncertainties are \pm 0.1 and \pm 0.05 a. u., respectively. The major uncertainty is in the transverse momentum of the recoil ion that arises solely from the temperature of the cooled beam due to its Maxwellian velocity distribution. The $\mathrm{He}$ target beam has a temperature of approximately $1 \mathrm{~K}$. This translates to an $\mathrm{x}$ - and $\mathrm{y}$-momentum one standard deviation uncertainty of 0.15 a. u., for a total of 0.22 a. u. in the transverse direction.

In the CTMC calculations this uncertainty has been including by means of a Monte Carlo scheme. At the end of each trajectory, after the system has reached its 
steady final state, the electron and the recoil momenta are randomly perturbed following a normal distribution of probability. Then, the momentum transfer $\boldsymbol{Q}$ and electron scattering angle are calculated from these perturbed momenta. Equivalently, within the CDW theory the perpendicular component of the momentum transfer has been smeared with a normal distribution of probability of width equal to $0.22 \mathrm{a}$. u. No integrations over the electron energy or angle have been performed in the CDW calculations, unlike in the CTMC case.

The cross sections are found to be strongly modified by the inclusion of the experimental momentum uncertainty and resolution (Fig. 3). Not only do the shapes of the TDCS's change, but also their absolute values are significantly increased by almost an order-of-magnitude at intermediate values of momentum transfer. We note that previous calculations show discrepancies in magnitude with experiment by factors of one to two orders-of-magnitude [6], while the present results are presented on the same absolute scale. It is clear that in our calculations the observed cross sections are mainly determined by the behavior for small $Q_{\text {perp }}$. Larger values of the momentum transfer do not contribute appreciably because the TDCS decreases rapidly. Thus, the shape of the angular distributions is mainly determined by the component of small $Q_{\text {perp }}$ observed in the experiment. As a result, the angular distributions are similar for all values of momentum transfer; such has been the trend observed in the experimental data [6]. The explanation for this behavior lies in the transverse momentum uncertainty of the recoil ion, which has a single standard deviation uncertainty of $0.22 \mathrm{a}$. u. The magnitude of the cross sections is steeply decreasing with increasing transverse momentum transfer. Thus, the small momentum transfer cross sections largely determine the cross sections even when they are two to three standard deviations away from the central momentum transfer value. 


\section{Effect of two electrons and electron correlation}

Good agreement is observed between the three-body classical and quantum-mechanical theories shown in the previous section. Moreover, the inclusion of the experimental uncertainties into the calculations decreases the large gap between calculated and measured cross sections magnitudes observed in a previous work [6]. However, none of the present approaches are able to accurately reproduce the shape of the experimental data.

One possible reason for the discrepancy is the use of three-body models in all theories. In order to test the four-body nature of the problem we performed $d$-CTMC calculations that explicitly include the two target electrons. In the previous three-body theories the projectile interacts with only two centers, namely, the active electron and the residual nucleus, while the effect of the passive electron is included as a modification to the nuclear Coulomb potential in a central mean field approximation. On the other hand, the $d$-CTMC models employed here fully consider the projectile dynamics. The deflection of the projectile is the result of the interactions with the three target particles, not a time-averaged $\mathrm{He}^{+}$core potential with a single electron. Moreover, the loss of flux due to double ionization is included.

Shown in Fig. 4 are the CTMC triple differential cross sections for ionization of $10 \mathrm{eV}$ electrons for a projectile transfer of 0.65 a.u. The four body calculations result in wider distributions than found for the three-body CTMC case, with the maximum of the distributions shifted towards the forward direction. These differences from the 3body results are most likely due to the dynamical nature of the projectile-core interaction which samples specific, not time averaged, regions of interaction space between the projectile and the two electrons.

Major differences still exist between our 3- and 4-body theories and 
experiment, in particular the lack of the observed strong focussing of the electrons towards zero degrees. Since all our calculations include the post-collision interaction (PCI) between projectile and electron, this focussing cannot be attributed to a PCI effect. In another paper [10] we show that there is a strong forward enhancement of the electrons if the scattering plane is tilted by approximately $30^{\circ}$. The shapes of the cross sections are then very similar to the experimental data. However, it is not for us to conclude that there may be a systematic error in the analysis of the data.

\section{Conclusions}

The triple differential cross sections have been investigated for single ionization of helium atoms by impact of highly charged ions at an incident velocity of 12 a.u. Comparison of results obtained by the $\mathrm{CDW}$ and CTMC methods has permitted us to determine some of the origins of the characteristics of the cross sections observed in recent experiments.

Within the CTMC theory, three different approaches were presented. In addition to a conventional three-body model, two different 4-body models were employed. These latter models allow one to study the effect of the correlation between the two target electrons along with a realistic incorporation of the loss of flux to the double ionization channel.

The agreement of the unaveraged quantal and classical theories with available experimental data is poor. However, the event-by-event evaluation of the momenta in the CTMC method allows us to introduce the experimental momentum uncertainties into the calculations. Inclusion of the experimental acceptances greatly changes the calculated cross sections. When the experimental momentum uncertainties are included, the fully differential cross section magnitudes change by over an order-of-magnitude from the 
unaveraged results and show general agreement with the measurements. However, as of yet no calculations have been able to reproduce the forward peaking of the TDCS's for the $3.6 \mathrm{MeV} / \mathrm{u} \mathrm{Au}^{53+}$ system.

\section{Acknowledgements}

Work supported by the Office of Fusion Energy Sciences, U. S. DOE. Work performed by UC-LLNL was performed under the auspices of the U.S. DOE under contract W7405-Eng-48 


\section{References}

[1] R. E. Olson, J. Fiol, J. Phys. B 34 (2001) L625.

[2] R. Moshammer, A. Perumal, M. Schulz, V. D. Rodríguez, H. Kollmus, R. Mann, S. Hagmann, J. Ullrich, Phys. Rev. Lett. 87 (2001) 223201.

[3] J. Fiol, R. E. Olson, J. Phys. B 35 (2002) 1759.

[4] J. Fiol, R. E. Olson, Nucl. Instrum. Methods B 205 (2003) 474.

[5] M. Schulz, R. Moshammer, D. H. Madison, R. E. Olson, P. Marchalant, C. T.

Whelan, H. R. J. Walters, S. Jones, M. Foster , H. Kollmus, A. Cassimi, J. Ullrich, J. Phys. B 34 (2001) L305.

[6] D. Fischer, R. Moshammer, M. Schulz, A. Voitkiv, J. Ullrich, Phys. B 36 (2003) 3555.

[7] W. Schmitt, R. Moshammer, F. S. C. O’Rourke, H. Kollmus, L. Sarkadi, R. Mann, S. Hagmann, R. E. Olson, J. Ullrich, Phys. Rev. Lett. 81 (1998) 4337.

[8] V. D. Rodríguez, Nucl. Instrum. Methods B 205 (2003) 498.

[9] J. Fiol J, V. D. Rodríguez, R. O. Barrachina, J. Phys. B 34 (2001) 933.

[10] J. Fiol, R. E. Olson, J. Phys. B in press (2004).

[11] R. H. Garvey, C. H. Jackman, A. E. S. Green, Phys. Rev. A 12 (1975) 1144.

[12] D. J. W. Hardie, R. E. Olson, J. Phys. B 16 (1983) 1983.

[13] C. J. Wood, C. R. Feeler, R. E. Olson, Phys. Rev. A 56 (1997) 3701.

[14] R. Moshammer, private communication (2003). 


\section{Figure Captions}

1. Continuum distorted wave triply differential cross sections (TDCS) for single ionization of $\mathrm{He}$ by impact of $3.6 \mathrm{MeV} / \mathrm{u} \mathrm{Au}^{53+}$. The triple differential cross-sections $\mathrm{d} \sigma / \mathrm{dE}_{\mathrm{el}} \mathrm{d} \Omega_{\mathrm{el}} \mathrm{d} Q_{\text {perp }}$ are presented as a function of the electron energy $\mathrm{E}_{\mathrm{e}}$ and $Q_{\text {perp }}$, the component of the momentum transferred by the projectile to the target system in the direction perpendicular to the incident velocity $v$, atomic units are used for all cross sections. The electron is emitted with $10 \mathrm{eV}$ of energy in the scattering plane. No averaging over experimental conditions has been made.

2. Comparison of CDW and CTMC triple differential cross sections for He ionization by

3.6 MeV/u Au${ }^{53+}$. The results were averaged over acceptance bins similar to those reported for the recent experiments $[2,6]$; namely $\mathrm{E}_{\mathrm{el}}=10 \pm 3 \mathrm{eV}, \theta_{\mathrm{el}}= \pm 5^{\circ}$, and $\varphi_{\mathrm{el}}= \pm$ $10^{\circ}$. The solid squares correspond to experimental data from Fischer et al [6].

3. TDCS for single ionization of helium. Electrons are emitted into the scattering plane with an energy of $10 \mathrm{eV}$. The solid squares correspond to experimental data from Fischer et al [6]; open symbols connected by lines are 3-body CTMC results and the solid lines are the CDW calculations. The calculations are similar to those of Fig. 2 with the addition that the theoretical results have been convoluted with the experimental momenta uncertainties assuming a normal probability distribution as explained in the text.

4. Comparison of three- and four-body CTMC TDCS's for a momentum transfer of $Q=0.65$ a. u. and emission of $10 \mathrm{eV}$ electrons into the scattering plane. The cross sections have been averaged by the experimental conditions as in Fig. 3. 


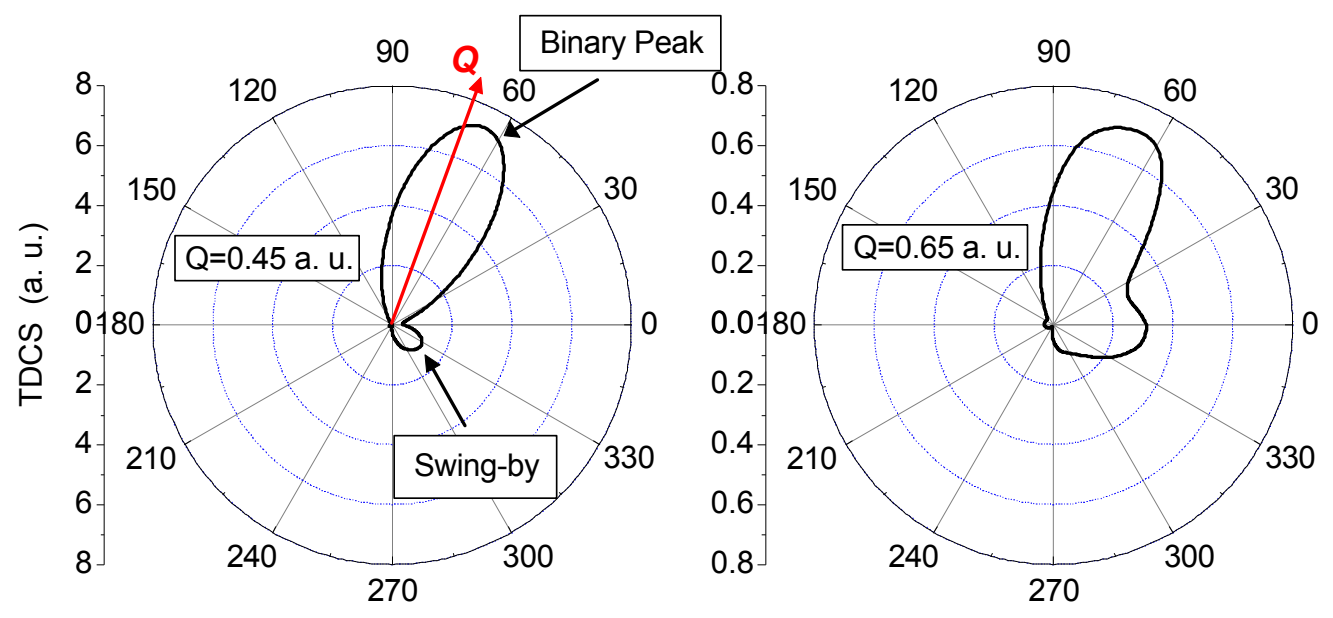

Figure 

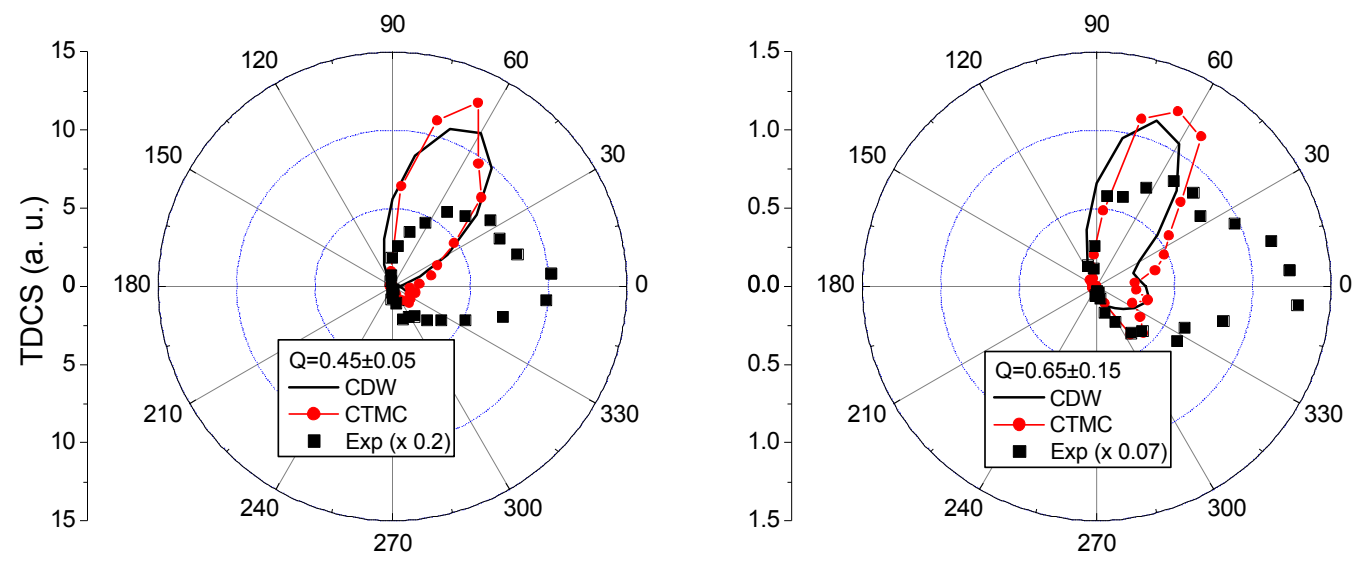

Figure 

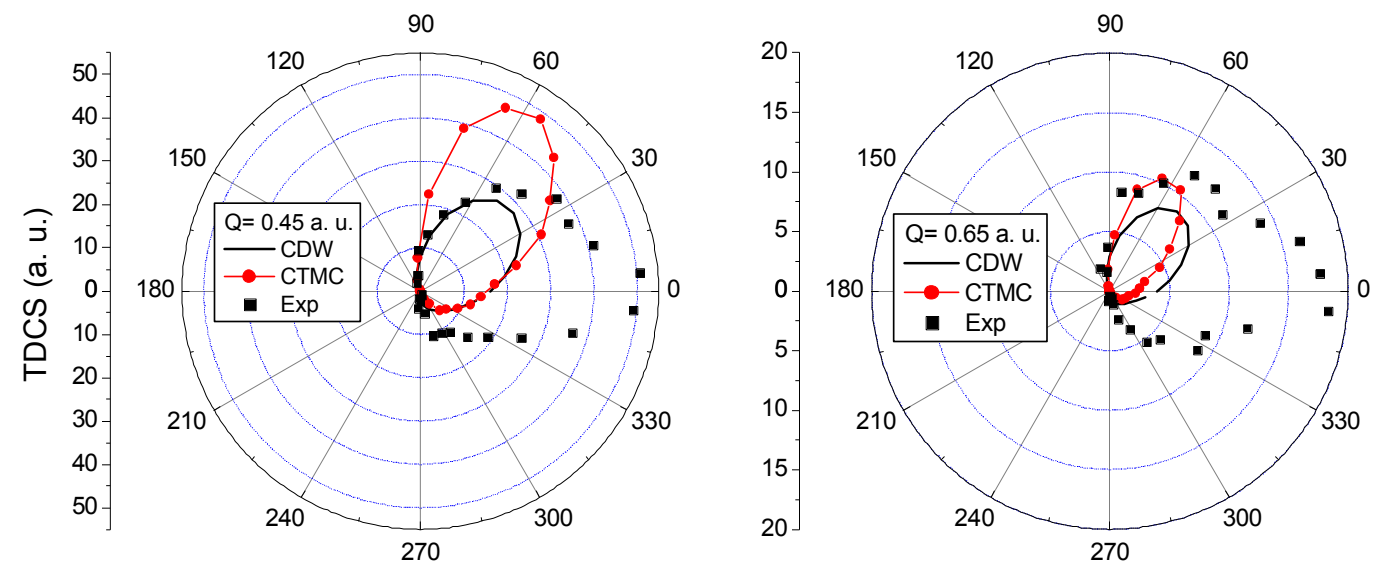

Figure 


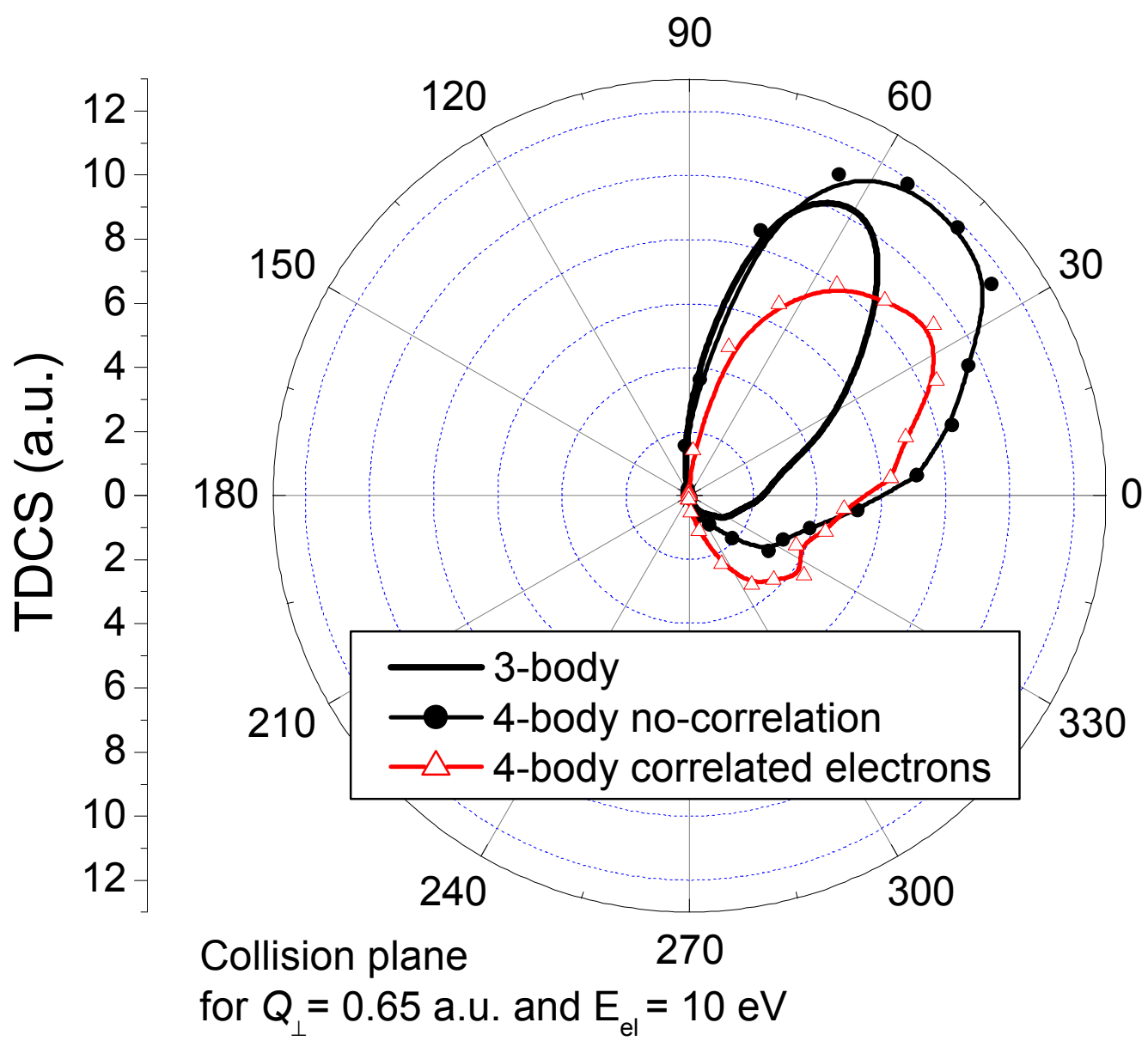

Figure 4 